\title{
New Findings Highlight Potential Risks of Common Drug Combination in Cardiac Patients
}

David N. Juurlink, Tara Gomes and Muhammad M. Mamdani

\section{The Issue}

Clopidogrel (Plavix) is a commonly used medication in patients with cardiovascular disease. It works by interfering with the activity of platelets (blood elements responsible for clotting) and is often combined with acetylsalicylic acid (ASA) in patients who have had a myocardial infarction or stent. It is also used in selected patients with stroke. In 2007, clopidogrel was the second leading drug worldwide, with more than US\$7.3 billion in sales, which is not surprising given the prevalence of cardiovascular and cerebrovascular diseases.

Scientists have known for some time that clopidogrel is a prodrug - that is, it has no effect on platelets until it is converted by the liver into its active form. The liver's ability to "turn on" clopidogrel is determined, in part, by genetics. Some patients (particularly those of Asian descent) lack the enzyme that converts clopidogrel to its active form. Recently, however, scientists have observed that some drugs can also interfere with this same process by blocking the primary liver enzyme responsible for this crucial step in the activation of clopidogrel.

Of the drugs that could interfere with the activation of clopidogrel, by far the most important are the proton pump inhibitors (PPIs). These drugs are extremely popular, and millions of Canadians take one of these drugs for a variety of common acid-related disorders, including gastroesophageal reflux disease and peptic ulcer disease, and as a preventative measure against stomach injury from anti-inflammatory drugs such as ASA. Given the popularity of PPIs and the large number of patients treated with clopidogrel, it is likely that millions of patients take these drugs in combination worldwide.

Some PPIs, but not others, interfere with the activity of the enzyme responsible for converting clopidogrel to its active form. Several laboratory-based studies have shown that certain PPIs appear to reduce the effect of clopidogrel in platelets, making blood "stickier" and more likely to clot. These findings support the notion that, in some patients, PPIs could reduce the effect of clopidogrel and increase the risk of cardiac events. However, until recently, no published studies characterized the clinical significance of the drug interaction between clopidogrel and PPIs. A recent study conducted at ICES and funded in part by the Ontario Ministry of Health and Long-Term Care provided the first large-scale insight into the clinical consequences of this extremely common drug interaction (Juurlink et al. 2009).

\section{Investigators estimate that between}

7 and $14 \%$ of all recurrent myocardial infarctions in their sample might result from the simultaneous use of clopidogrel and certain PPIs.

\section{The Findings}

Key findings of the study were as follows (Juurlink et al. 2009):

- The authors identified 13,636 older Ontario residents who were started on clopidogrel following acute myocardial infarction over a period of nearly six years. Of these, 734 were readmitted with another myocardial infarction within 90 days.

- Treatment with any PPI in addition to clopidogrel was associated with a $27 \%$ increase in the risk of a recurrent myocardial infarction relative to clopidogrel alone, but no increase in the risk of death.

- When the PPIs were grouped according to their effects on the liver enzyme that converts clopidogrel to its active form, certain PPIs that inhibit that process (omeprazole, lansoprazole and rabeprazole) were associated with a $40 \%$ increased risk of a recurrent myocardial infarction, whereas pantoprazole (which does not inhibit the enzyme) showed no such effect. However, this was a secondary analysis, and further research is needed to confirm the safety of pantoprazole in patients receiving clopidogrel.

- Importantly, the combination of PPIs and clopidogrel was extremely common. Almost one third of clopidogrel-treated patients were taking a PPI within 90 days of discharge after a myocardial infarction. Because of the high co-prescription rate, the investigators estimate that between 7 and $14 \%$ of all 
recurrent myocardial infarctions in their sample might result from the simultaneous use of clopidogrel and omeprazole, lansoprazole or rabeprazole.

\section{Conclusions and Implications}

This study documents the real-world consequences of an extremely common drug interaction that, until now, has been speculative and based on pharmacology theory and laboratory studies. The findings were recently confirmed by another study from the United States (Ho et al. 2009), and other similar publications are anticipated in the future.

The clinical implications of this research suggest that physicians should be more selective in their use of PPIs in patients who are receiving clopidogrel. For many patients, older acid-lowering drugs such as ranitidine may be suitable and will not increase the risk of recurrent cardiac events. For patients in whom a PPI and clopidogrel are essential, clinicians should consider limiting the dose and duration of therapy. A secondary analysis of this study suggests that pantoprazole may be the preferred agent in this setting since there is, at present, no evidence that it interferes with the activity of clopidogrel. Future research is necessary to explore the relative safety of individual PPIs in patients receiving clopidogrel. $\mathrm{HQ}$

\section{Acknowledgements}

This study was supported by the Ontario Ministry of Health and Long-Term Care and by the Canadian Institutes of Health Research.

\section{References}

Ho, P.M., T.M. Maddox, L. Wang, S.D. Finn, R.L. Jesse, E.D. Peterson and J.S. Rumsfeld. 2009. "Risk of Adverse Outcomes Associated with Concomitant Use of Clopidogrel and Proton Pump Inhibitors following Acute Coronary Syndrome. Journal of the American Medical Association 301(9): 937-44.

Juurlink, D.N., T. Gomes, D.T. Ko, P.E. Szmitko, P.C. Austin, J.V. Tu, D.A. Henry, A. Kopp and M.M. Mamdani. 2009. "A Population-Based Study of the Drug Interaction between Proton Pump Inhibitors and Clopidogrel." Canadian Medical Association Journal 180(7): 713-18.

\section{About the Authors \\ David N. Juurlink, BPhm, MD, PhD, FRCPC, is a scientist at the Institute for Clinical Evaluative Sciences (ICES) in Toronto, Ontario. He is also head of the Division of Clinical Pharmacology and Toxicology at Sunnybrook Health Sciences Centre and associate professor of medicine, pediatrics and health policy, management and evaluation at the University of Toronto.}

Tara Gomes, MHSc, is an epidemiologist with the Ontario Drug Policy Research Network at ICES.

Muhammad M. Mamdani, PharmD, MA, MPH, is director of the Applied Health Research Centre of the Li Ka Shing Knowledge Institute of St. Michael's Hospital in Toronto, Ontario. He is also an adjunct scientist at ICES and associate professor of medicine, pharmacy and health policy, management, and evaluation at the University of Toronto.

\title{
a journal with a distinguished history
}

\author{
W ORLD HEALTH \& POPULATION
}

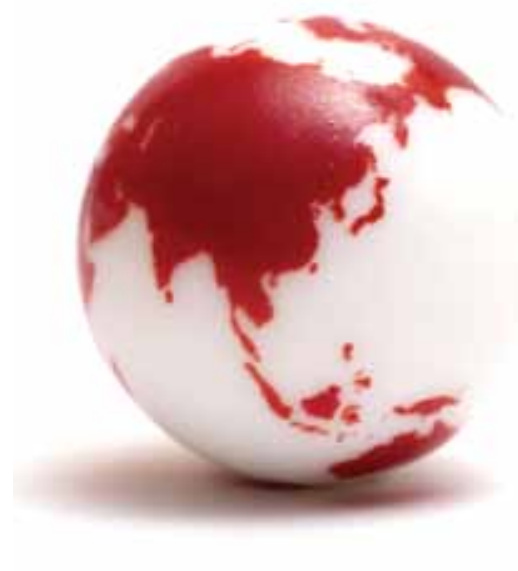

www.worldhealthandpopulation.com 